\title{
Gut Microbiota and Ischemic Stroke: The Role of Trimethylamine N-Oxide
}

\author{
Hyo Suk Nam \\ Department of Neurology, Yonsei University College of Medicine, Seoul, Korea
}

Trimethylamine N-oxide (TMAO) is produced when trimethylamine, a waste product of gut microbes, is converted via hepatic flavin monooxygenases. As TMAO is a potential causative factor in various cardiovascular diseases (CVDs) considerable research interest has arisen on its use as a biomarker. Higher TMAO levels are associated with future risk of both incident CVD in the general population and established CVD, including stroke. The addition of TMAO into models with traditional risk factors significantly improved the prediction of future CVD risk. TMAO promotes atherosclerosis and is associated with platelet hyperreactivity and inflammation, which are in turn associated with the development of stroke and its secondary consequences. Additionally, TMAO may play a key mediator role in the relationship between the diet, gut microbiota, and CVD development. Compelling evidence suggesting that TMAO is both a risk factor and prognostic marker of stroke and CVD. Potential therapeutic strategy of diet and drugs in reducing TMAO levels have emerged. Thus, TMAO is a novel biomarker and target in stroke and CVD prevention.

\author{
Correspondence: Hyo Suk Nam \\ Department of Neurology, Yonsei \\ University College of Medicine, 50-1 \\ Yonsei-ro, Seodaemun-gu, Seoul 03722, \\ Korea \\ Tel: $+82-2-2228-1617$ \\ Fax: +82-2-393-0705 \\ E-mail: hsnam@yuhs.ac \\ Received: February 28, 2019 \\ Revised: April 5, 2019 \\ Accepted: April 18, 2019
}

Keywords Trimethylamine N-oxide; Stroke; Prognosis

\section{Introduction}

An increasing body of evidence suggests that the human intestinal gut microbiota-composed of tens of trillions of bacteriaand microbiota-derived metabolites play roles in various diseases, including cardiovascular disease (CVD) and stroke. ${ }^{1}$ The human microbiota includes at least 1,000 species of known bacteria harboring more than 3 million genes (150 times more genes than the human genome). The brain and gut are connected by a network of neurons, forming a complex microbiota-gut-brain axis that exhibits strong bi-directional interactions.

Accumulating evidence also suggests that the intestinal microbiota plays an important role in the pathophysiology and outcome of stroke. Ischemic stroke alters the composition of the intestinal microbiota. Conversely, the intestinal microbiota can modulate the outcome of stroke and plays a role in the pathogenesis of stroke. Clinical and experimental studies have reported that the gut microbiota is associated with risk factors for stroke such as hypertension, diabetes, and obesity. ${ }^{2}$

Clinically, risk stratification for stroke remains a challenge. Clinical scoring algorithms have been developed to aid in the prediction of adverse events. Biomarkers can provide additional clinically useful information. The interest in combining clinical and biochemical markers for use in precision medicine is therefore growing ${ }^{3,4}$ and metabolomics is a relatively new and promising technology for identifying useful biomarkers. ${ }^{5}$

Trimethylamine $\mathrm{N}$-oxide (TMAO) is a metabolite generated as a waste product of the gut microbiota which is associated with both CVD and stroke. Increased TMAO levels have been linked to increased risk of incident major adverse cardiovascular events (MACEs) independent of traditional cardiovascular risk factors. Moreover, TMAO is directly linked to poor out- 
comes in patients with CVD, ${ }^{6,7}$ Despite numerous studies demonstrated its harmful effects, several studies have suggested that TMAO plays a protective role. Other studies have suggested that TMAO is a marker of a disruption in homeostasis rather than a causative or protective factor. ${ }^{8,9}$ This review will discuss the current research on the production of TMAO, its association with CVD and stroke and related pathogenic mechanisms, analytical methods, and potential therapeutic strategies in addition to areas of continued debate.

\section{Production of TMAO}

TMAO is formed via a two-step process, the meta-organismal diet/microbiota dependent pathway and human endogenous pathway. In the first step, trimethylamine (TMA) is generated from the dietary nutrients (e.g., choline, phosphatidylcholine, Lcarnitine) by the activity of gut microbiota TMA lyases. In the second step, TMA is absorbed and oxidized to TMAO by hepatic flavin-containing monooxygenases (FMOs). The specific activity of FMO3 for oxidizing TMA is $>10$ times that of $\mathrm{FMO} 1 .{ }^{10} \mathrm{FMO} 3$ plays an important role in the regulation of blood glucose and cholesterol levels in addition to bile acid metabolism. ${ }^{11}$ Fish odor syndrome (trimethylaminuria) is a genetic disease associated with mutations in the $\mathrm{FMO} 3$ gene. Loss-of-function mutations in FMO3 lead to increased levels of volatile TMA, which has a fish-like odor (Figure 1). ${ }^{9}$

The TMA-containing nutrients choline, phosphatidylcholine, and L-carnitine are subsequently degraded by a TMA lyase produced by specific intestinal bacteria. The microbiota plays a critical role in TMA formation, as evidenced by antibiotic knockdown studies clearly demonstrating that TMAO is not formed in the absence of a microbiota. ${ }^{7}$ Microbial transplantation studies showed that potential TMAO production is a transmissible trait linked with atherosclerosis and thrombosis, suggesting that TMAO is a factor in atherogenesis and thrombosis risk. ${ }^{12}$ Although specific members of the gut microbiota involved in TMA generation have not been identified, previous studies have reported a relationship between plasma TMAO levels and members of the phylum Tenericutes and genus Desulfovibrio. The general function of TMAO in mammals has yet to be elucidated; however, in saltwater fish and crustaceans, TMAO serves as a major osmolyte and chaperone that stabilizes the protein structure, countering the denaturing effects of urea and high ambient water pressure. ${ }^{13}$

\section{Diet and TMAO}

Diet profoundly affects the gut microbiota and can alter the overall bacterial composition. ${ }^{14}$ High consumption of meat, choline, and L-carnitine increases the formation of TMAO. Phosphatidylcholine is a major dietary source of choline commonly found in the Western diet such as red meat, eggs, and other meat products. ${ }^{15}$ In healthy volunteers given a phosphatidylcholine challenge before and after antibiotic-mediated suppression of the gut microbiota, choline metabolites increased after the phosphatidylcholine challenge. The antibiotic treatment suppressed TMAO generation and TMAO formation resumed when the antibiotics were withdrawn. These data indicate that dietary phosphatidylcholine intake is associated with increased TMAO levels.?

Significant diet-host interactions also affect TMAO production. Inter-individual variations in circulating and urinary TMAO concentrations have been reported following the consumption of eggs and choline supplements. ${ }^{16,17}$ Additionally, blood TMAO concentrations increased after a high-fat diet. ${ }^{18}$

\section{TMAO and CVD}

The results of recent clinical studies suggest a correlation between elevated plasma TMAO levels and the risk of stroke, atrial fibrillation, diabetes, congestive heart failure and chronic kidney, coronary artery, and peripheral artery disease. TMAO is also an independent and dose-dependent risk factor for CVD, and elevated systemic TMAO concentrations are correlated with an increased future risk of cardiovascular events and allcause mortality.

\section{TMAO and stroke}

Relatively few studies have addressed the relationship between TMAO and stroke. A nested case-control study of a hypertensive Chinese population showed that higher TMAO levels were associated with an increased risk of first stroke. Patients in the upper tertiles had a 34\% higher risk of first stroke than those in the lowest tertiles. They also found that patients with low folate and high TMAO had the highest rate of stroke..$^{19}$ In patients with firstever stroke, elevated TMAO levels exhibit a dose-dependent association with the risk of recurrent stroke and subsequent cardiovascular events. This relationship remains even after adjusting for traditional cerebrovascular risk factors and initial stroke severity. Blood TMAO concentration is closely related to the number of proinflammatory intermediate CD14++/CD16+ monocytes. ${ }^{12}$

A case-control study of Chinese patients with stroke and transient ischemic attack (TIA) showed significant dysbiosis of the gut microbiota. Importantly, stroke and TIA patients exhibited lower plasma TMAO concentrations than control patients with asymptomatic atherosclerosis. The authors explained that 


\section{Phosphatidylcholine}

\section{Choline Carnitine}
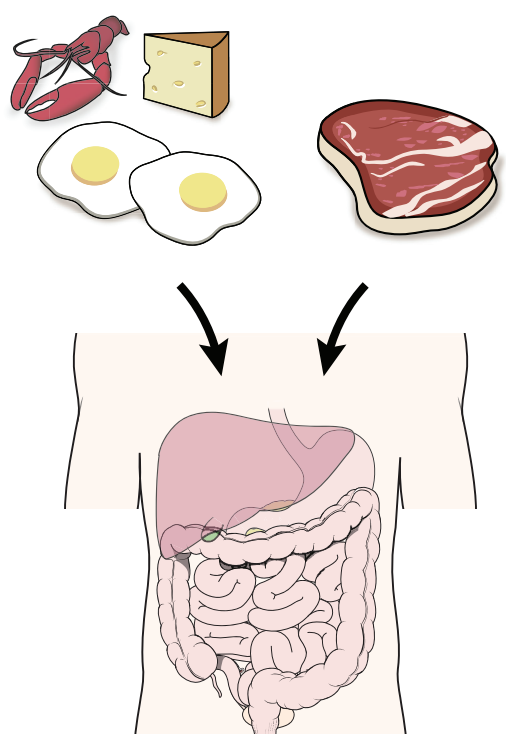<smiles>C[N+](C)(C)CC(O)CC(=O)O</smiles>

\section{L-Carnitine}

\section{Gut microbiota} (large bowel)

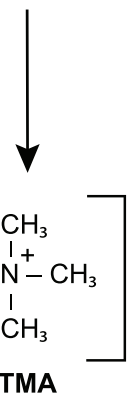

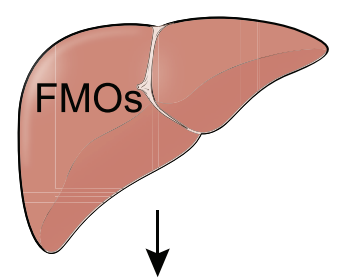

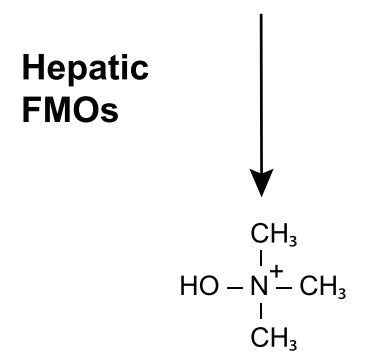

TMAO

TMAO

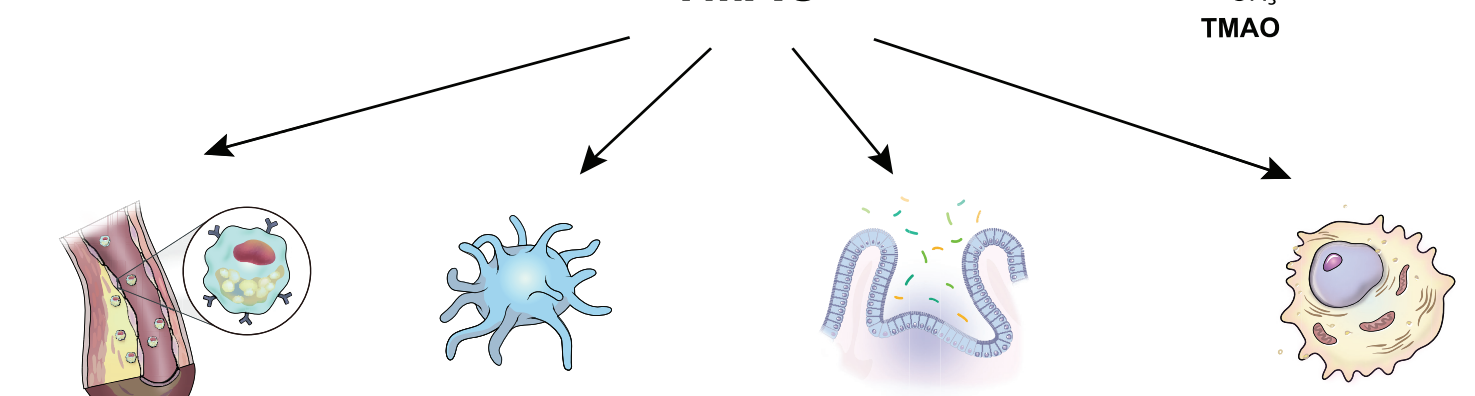

Foam cell formation

Platelet hyperactivity
Altered bile acids and cholesterol transport

\section{Inflammation}

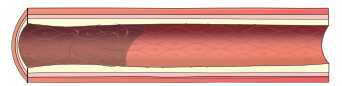

Thrombosis

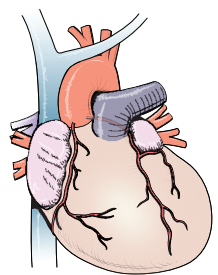

Heart failure

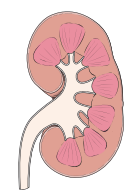

Chrnoic kidney disease

Figure 1. Production of trimethylamine N-oxide (TMAO) and association with atherosclerosis and thrombosis resulting in cardiovascular diseases. Trimethylamine (TMA) is generated by the action of TMA lyases in the gut microbiota from dietary TMA-containing nutrients (e.g., choline, phosphatidylcholine, L-carnitine). Then, TMA is rapidly further oxidized to TMAO by hepatic flavin-containing monooxygenases (FMOs). TMAO promotes macrophage foam cell formation, development of platelet hyperreactivity, altered bile acids and cholesterol transport, and activation of the inflammatory pathway. All factors are associated with an increased risk of cardiovascular diseases including stroke, myocardial infarction, congestive heart failure, and chronic kidney disease. 
they examined the TMAO level in patients who already had stroke or TIA, the level of TMAO was quite low compared to a previous Western study, and the treatment of stroke or TIA may reduce the TMAO levels. ${ }^{20}$

A multicenter study reported that plasma TMAO levels before carotid artery stenting were significantly higher in patients with new lesions on post-stenting diffusion-weighted image (DWI) than in patients without new lesions. After adjusting for possible confounders, elevated plasma TMAO levels remained an independent predictor of new lesions on DWI after carotid artery stenting. ${ }^{6}$ Moreover, increased serum TMAO levels have been associated with increased carotid intima-media thickness in subjects at risk for type 2 diabetes, independent of insulin resistance, visceral obesity, and fatty liver. Following a lifestyle modification intervention, the carotid intima-media thickness significantly decreased in subjects who exhibited the greatest decrease $(>20 \%)$ in TMAO levels. ${ }^{21}$

\section{TMAO and atrial fibrillation}

A recent study demonstrated a positive association between plasma TMAO levels and long-term incident atrial fibrillation in patients with suspected stable angina. This finding was validated in a community cohort of elderly participants. ${ }^{22}$

\section{TMAO and diabetes}

Patients with diabetes typically have higher TMAO concentrations, MACEs, and mortality risk independent of traditional risk factors, renal function, or glycemic control than the healthy controls. ${ }^{23}$ In contrast, a case-control study showed that patients with high baseline TMAO levels had a lower risk of developing diabetes after adjustments were made. ${ }^{24}$

\section{TMAO and coronary artery disease}

Over a 3-year follow-up period of patients who underwent elective coronary angiography, the plasma TMAO concentrations were positively associated with the rate of MACEs. Patients in the highest quartile of circulating TMAO level exhibited a 2.5-fold higher risk of MACEs than patients in the lowest quartile, even after adjustment for traditional risk factors. ${ }^{7}$ In acute myocardial infarction, TMAO concentrations were also associated with a poor prognosis (death/myocardial infarction) at 2 years (hazard ratio [HR], 1.21; 95\% confidence interval [Cl], 1.03 to 1.43) but was not able to predict death/myocardial infarction at 6 months ( $\mathrm{HR}, 1.19 ; 95 \% \mathrm{Cl}, 0.96$ to 1.48). ${ }^{3}$

An optical coherence tomography study of patients with acute coronary syndrome demonstrated that TMAO levels were associated with the vulnerability and progression of coronary plaque and the long-term risks of cardiovascular events in pa- tients with acute coronary syndrome..$^{25}$ Plasma TMAO concentrations were significantly higher in patients with ST-segment elevation myocardial infarction with plaque rupture than in patients exhibiting plaque erosion. The authors suggested that TMAO could be a useful biomarker for predicting plaque rupture in patients with a history of acute myocardial infarction. ${ }^{26}$

\section{TMAO and heart failure}

Data also suggest that high TMAO levels are associated with the development and progression of heart failure. Patients with stable heart failure have higher TMAO levels than the healthy subjects. In patients with acute heart failure, TMAO was found to be associated with in-hospital mortality and poor prognosis at 1 year. The authors suggest that both TMAO and N-terminal pro-brain natriuretic peptide provide additional prognostic information. ${ }^{27}$ Interestingly, following the adjustment for renal function markers such as the estimated glomerular filtration rate and urea, TMAO was no longer significantly associated with these outcomes. ${ }^{28}$ Patients with chronic heart failure have elevated TMAO levels, which are positively correlated with the disease severity and adverse outcomes during follow-up. ${ }^{29} \mathrm{Ad}-$ ditionally, these patients with elevated circulating TMAO levels have a poorer prognosis at 5 years. ${ }^{30}$

\section{TMAO and chronic kidney disease}

Patients with chronic kidney disease exhibit increased blood TMAO concentrations, and this is inversely associated with the glomerular filtration rate. Elevated TMAO levels are strongly associated with decreased renal function. Dialysis can effectively remove TMAO from the circulation in patients with elevated TMAO levels, and TMAO levels were within normal range after renal transplantation. ${ }^{31,32}$ In patients with advanced chronic kidney disease, an elevated TMAO level was found to be independently associated with MACE risk after adjustment for cardiovascular risk factors. ${ }^{32,33}$

Studies examining the relationship between TMAO levels and cardiovascular outcomes have identified kidney function as an important confounder. Patients with CVD typically exhibit a declining kidney function, which in turn is a well-established risk factor for exacerbation of CVD. Elevated TMAO levels are thought to be indicative of renal medullary damage resulting from CVD-associated hypertension. ${ }^{17}$

\section{TMAO and peripheral arterial obstructive disease} TMAO was found to be a significant predictor of a 5-year allcause mortality risk among patients with stable peripheral artery diseases, including lower extremity peripheral artery disease and renal, mesenteric, and carotid artery stenosis. When 
TMAO was included as a covariate in risk analyses, a significant improvement in the accuracy of risk estimations was noted..$^{15}$

\section{TMAO and Alzheimer's disease}

The gut microbiota can influence the aging process and cognitive dysfunction. Plasma TMAO levels increased in both the elderly and aged mice. TMAO promotes neuronal senescence, synapse damages, and reduces the synaptic plasticity. ${ }^{34} \mathrm{~A}$ recent study showed that cerebrospinal fluid TMAO levels were elevated in individuals with Alzheimer's disease (AD), and an elevated cerebrospinal fluid TMAO level is associated with elevated $A D$ pathology and neuronal degeneration. TMAO may play a role in $A D$ pathology by promoting cerebrovascular disease as a vascular risk factor is common in AD patients, and cerebrovascular pathology frequently coexists with $A D$ pathology. ${ }^{35}$

\section{Mechanism of TMAO associated atherosclerosis and thrombosis}

The mechanism by which TMAO promotes atherosclerosis is poorly understood. Several mechanisms have been proposed, including promotion of foam cell formation via TMAO-mediated increased expression of scavenger receptors on macrophages, changes in cholesterol, sterol, bile acid metabolism, sterol transporters, and activation of pro-inflammatory pathways. ${ }^{25}$

\section{Foam cell formation}

Wang et al. ${ }^{36}$ were the first to report a potential mechanistic link between TMAO and development of atherosclerosis. They reported that TMAO promotes macrophage foam cell formation that is associated with increased expression of two scavenger receptors of CD36 and scavenger receptors class A1 (SRA1). ${ }^{36}$ Another group reported that the susceptibility to atherosclerosis can be transferred via transplantation of gut microbes in a mouse model. ${ }^{37}$ Suppression of TMAO generation using a small-molecule inhibitor of microbial TMA production inhibits atherosclerosis, and animal model studies demonstrated that suppression of FMO3 activity leads to both a reduction in TMAO levels and inhibition of atherosclerosis."

\section{Platelet hyperreactivity and thrombosis}

Studies conducted on animal models and healthy volunteers indicated that TMAO contributes directly to platelet hyperreactivity and increases the risk for thrombosis. Sub-maximal stimulusdependent platelet activation by multiple agonists was enhanced by direct exposure of the platelets to TMAO in a process mediated by an augmented release of $\mathrm{Ca}^{2+}$ from intracellular stores. TMAO-mediated modulation of platelet hyperreactivity and thrombosis potential was confirmed in a microbial transplantation study using the germ-free mice. These data suggest that therapies targeting TMAO could have beneficial anti-thrombotic effects without increased risk of bleeding complications. ${ }^{38}$

\section{Cholesterol metabolism}

The bile acid pathway plays an important role in removing excess cholesterol from the circulation. Disruption of normal bile acid synthesis and metabolism has been linked to an increased risk of atherosclerosis. TMAO plays a significant role in cholesterol metabolism in the bile acid compartments, affecting the metabolism of cholesterol and sterol in macrophages, the liver and the intestines. A significant reduction in cholesterol absorption and hepatic expression of bile acid synthetic enzymes including cytochrome P450, family 7, subfamily a, polypeptide 1 (Cyp7a1) and cytochrome P450, family 27, subfamily a, polypeptide 1 (Cyp27a1) was noted. ${ }^{8}$ The mice which fed on a diet supplemented with TMAO, carnitine, or choline had decreased reverse cholesterol transport. Suppression of the intestinal microbiota by treatment with oral broad-spectrum antibiotics completely blocks the suppression of reverse cholesterol transport. ${ }^{39}$

\section{Inflammation}

Among the many deleterious effects of ischemic stroke, increased inflammation can lead to a substantial secondary damage following stroke. TMAO is known to activate the proinflammatory pathways and promote vascular inflammation and endothelial cell dysfunction through a variety of signaling pathways. TMAO functions as a switch that activates pro-inflammatory cascades, leading to arterial damage that allows the cholesterol to enter the arterial walls and subsequent plaque formation. A study using cultured human aortic endothelial and vascular smooth muscle cells reported that the expression of various inflammatory cytokines and adhesion molecules was upregulated by TMAO, mediated in part via the nuclear factor-KB signaling pathway. ${ }^{40,41}$

\section{Measurement of TMAO}

Plasma TMAO levels vary widely both within and between individuals. A number of factors affect the circulating TMAO levels, including age, diet, gut microbial flora, liver FMO enzymes, high-sensitivity C-reactive protein, and kidney function. Aside from the traditional protein-based biomarkers, there is an increased interest on the development of analysis methods for newer metabolite-based biomarkers, including TMAO. ${ }^{3}$ Liquid chromatography-tandem mass spectrometry is considered as the gold standard method for the quantification of TMAO, as it 
enables high-accuracy, -precision, and -throughput analyses suitable for the needs of clinical diagnostic practices. ${ }^{42}$

\section{A harmful, protective, or diagnostic marker?}

Although a considerable evidence suggests that TMAO plays a role in the development and pathogenesis of CVD, its precise role is still an issue of debate. Some studies have found no relationship between increased circulating TMAO levels and increased risk of MACEs, perhaps due to the differences in age, risk factors, geographic location, or dietary habits of the individuals in their respective cohorts.

For example, the aforementioned Chinese study demonstrated that patients with a history of large artery atherosclerotic stroke or TIA had lower TMAO levels than asymptomatic atherosclerotic patients. ${ }^{20}$ Similarly, a study conducted in Norway found no association between TMAO levels and carotid atherosclerosis or cardiovascular mortality. ${ }^{43} \mathrm{~A}$ population-based study of adults between 33 and 55 years reported that plasma TMAO was not associated with the 10-year incidence or progression of coronary artery calcium or intima-media thickness. ${ }^{44}$ Thus, these data suggest that TMAO may not be the "culprit" but may be considered as a potential biomarker. High levels of circulating TMAO may reflect changes in the composition of the gut microbiota which increase disease susceptibility. Accumulation of TMAO in a disease state may be caused by the adaptation of cells to stress. Thus, TMAO would represent a disease marker rather than a mediator. ${ }^{9}$

Although many researchers have recognized the harmful effects of TMAO, others have suggested that it plays a beneficial role. One group suggested that TMAO functions as a naturally occurring osmolyte, protecting cells against the harmful effects of various stressors, for example, by counteracting the denaturing activity of urea by enhancing the stability of cellular proteins. ${ }^{45}$ Furthermore, TMAO slows the formation of aortic atherosclerotic lesions in apolipoprotein E knockout mice. ${ }^{46}$ These data suggest that TMAO plays a role in preventing the development of atherosclerosis in humans.

Fish consumption is well known for its cardioprotective attributes in humans. However, it causes higher levels of circulating TMAO than consumption of either eggs or beef. One study reported that plasma TMAO levels increased within 15 minutes of consuming fish, which suggests that dietary TMAO is absorbed without processing by gut microbes. ${ }^{9}$ These conflicting data make it difficult to fully elucidate the relationship between CVD and dietary and gut microbe-mediated TMAO production. ${ }^{47}$

\section{Therapeutic strategy}

TMAO appears to be a novel and potentially modifiable risk factor for stroke. The potential roles played by diet and drugs in reducing TMAO levels have emerged.

Diet

Decreased dietary consumption of L-carnitine and choline has been shown to decrease TMAO levels..$^{39}$ Likewise, consumption of the Mediterranean or vegetarian diet reportedly aids in reducing TMAO production.

\section{Exercise}

Exercise can alter the microbiota diversity and distribution in humans. ${ }^{48}$ Voluntary exercise ameliorates obesity and metabolic disorders. An animal study showed that voluntary exercise normalizes the plasma TMAO levels and prevents cardiac dysfunction in western diet-induced obese mice. This effect was reversed by concomitant administration of TMAO and abrogated the beneficial effects. ${ }^{49}$

\section{Microbiota}

Several bacterial species that colonize the human gut have been shown to reduce TMAO levels. Thus, manipulation of the composition of commensal bacteria in the gut could be a novel therapeutic approach for preventing and treating CVD by modulating TMAO production. Therefore, probiotic formulations specifically tailored to this purpose could serve as the basis of a therapeutic strategy for treating CVD. ${ }^{36}$

\section{Antibiotics}

Healthy subjects treated with oral broad-spectrum antibiotics that induce suppression of the intestinal microbiota exhibited reduced plasma TMAO levels after the phosphatidylcholine challenge. The TMAO levels increased 1 month after cessation of antibiotic treatment. ${ }^{7}$ However, the potential undesirable side effects associated with the antibiotic treatment, in addition to the risk of the emergence of antibiotic-resistant bacteria make this approach less than the ideal.

\section{Aspirin}

Low-dose aspirin treatment attenuates both the degree of TMAO elevation and platelet hyperresponsiveness. ${ }^{50}$ The mechanisms by which aspirin exerts these effects have yet to be elucidated; however, it is thought that aspirin affects the composition of the gut microbiota. 


\section{FMO inhibitors}

Studies on animal models demonstrated that insulin or antisense oligonucleotide-mediated suppression of the hepatic enzyme FMO3 leads to a reduction in circulating TMAO levels and prevents hypercholesterolemia and atherosclerosis. FMO3 inhibition may be accompanied by several untoward side effects, including hepatic inflammation and noxious fish ordor. ${ }^{10,11}$

\section{Resveratrol}

Resveratrol (RSV) is a natural polyphenol found in grapes and berries. Compared with the control mice, markedly lower levels of serum TMA and TMAO were found in the mice treated with RSV and fed with choline. ${ }^{51}$

\section{Meldonium}

Meldonium, an aza-analogue of gamma-butyrobetaine (GBB), competes with GBB and L-carnitine and is the most potent substance commercially available for decreasing L-carnitine levels. The intestinal microbiota-dependent production of TMA/ TMAO from L-carnitine was significantly decreased following meldonium treatment. However, meldonium had no effect on either bacterial growth or bacterial uptake of L-carnitine. ${ }^{52}$

\section{3,3-d’ imethyl-1-butanol}

A 3,3-d'imethyl-1-butanol (DMB), which is a structural analogue of choline found in balsamic vinegar, red wines, and some olive oils and grape seed oils, inhibits TMA production by suppressing the activity of microbial TMA lyases. Moreover, it inhibits enhanced formation of endogenous macrophage foam cells and atherosclerotic lesions associated with consumption of a choline-rich diet. Consumption of DMB suppresses the growth of bacteria in taxa associated with increased levels of plasma TMA and TMAO. ${ }^{53}$

\section{Future work}

Most clinical studies on TMAO conducted recently have used a cross-sectional or cohort design, and few interventional studies have been reported. Thus, additional interventional studies are needed to determine with certainty the relationship between TMAO and CVD development and progression. Such studies could facilitate the development of a therapeutic strategy targeting TMAO.

\section{Conclusions}

Considerable evidence suggests that TMAO is an important risk factor and prognostic marker for stroke and CVD. Increased
TMAO generation promotes atherosclerosis, platelet activation, and inflammation. TMAO may be a central molecule in the relationship of diet, genetics, the gut microbiota, and CVD.

\section{Disclosure}

The author has no financial conflicts of interest.

\section{Acknowledgments}

The authors would like to thank the staff of the Medical Illustration \& Design, affiliated with the Medical Research Support Services of Yonsei University College of Medicine, for all artistic support related to this work.

This work was supported by the National Research Foundation of Korea (NRF) grant funded by the Korea government (MSIP) (2016R1C1B2016028).

\section{References}

1. Aron-wisnewsky J, Clement K. Dietary choline derived TMAO: new role in thrombosis. AME Med J 2017;2:112.

2. Winek $K$, Dirnagl U, Meisel A. Role of the gut microbiota in ischemic stroke. Neurol Int Open 2017;1:E287-E293.

3. Suzuki T, Heaney LM, Jones DJ, Ng LL. Trimethylamine N-oxide and risk stratification after acute myocardial infarction. Clin Chem 2017;63:420-428.

4. Kim SJ, Moon GJ, Bang OY. Biomarkers for stroke. J Stroke 2013;15:27-37.

5. Sidorov E, Sanghera DK, Vanamala JKP. Biomarker for ischemic stroke using metabolome: a clinician perspective. J Stroke 2019;21:31-41.

6. Wu C, Li C, Zhao W, Xie N, Yan F, Lian Y, et al. Elevated trimethylamine $\mathrm{N}$-oxide related to ischemic brain lesions after carotid artery stenting. Neurology 2018;90:e1283-e1290.

7. Tang WH, Wang Z, Levison BS, Koeth RA, Britt EB, Fu $X$, et al. Intestinal microbial metabolism of phosphatidylcholine and cardiovascular risk. N Engl J Med 2013;368:1575-1584.

8. Velasquez MT, Ramezani A, Manal A, Raj DS. Trimethylamine $\mathrm{N}$-oxide: the good, the bad and the unknown. Toxins (Basel) 2016;8:E326.

9. Cho CE, Taesuwan $\mathrm{S}$, Malysheva OV, Bender E, Tulchinsky NF, Yan J, et al. Trimethylamine-N-oxide (TMAO) response to animal source foods varies among healthy young men and is influenced by their gut microbiota composition: a randomized controlled trial. Mol Nutr Food Res 2017;61:1600324.

10. Miao J, Ling AV, Manthena PV, Gearing ME, Graham MJ, Crooke RM, et al. Flavin-containing monooxygenase 3 as a 
potential player in diabetes-associated atherosclerosis. Nat Commun 2015:6:6498.

11. Shih DM, Wang Z, Lee R, Meng Y, Che N, Charugundla S, et al. Flavin containing monooxygenase 3 exerts broad effects on glucose and lipid metabolism and atherosclerosis. J Lipid Res 2015;56:22-37.

12. Komaroff $A L$. The microbiome and risk for atherosclerosis. JAMA 2018;319:2381-2382.

13. Loscalzo J. Gut microbiota, the genome, and diet in atherogenesis. N Engl J Med 2013;368:1647-1649.

14. Ferguson JF, Allayee $H$, Gerszten RE, Ideraabdullah F, KrisEtherton PM, Ordovás JM, et al. Nutrigenomics, the microbiome, and gene-environment interactions: new directions in cardiovascular disease research, prevention, and treatment: a scientific statement from the American Heart Association. Circ Cardiovasc Genet 2016;9:291-313.

15. Senthong V, Wang Z, Fan Y, Wu Y, Hazen SL, Tang WH. Trimethylamine $\mathrm{N}$-oxide and mortality risk in patients with peripheral artery disease. J Am Heart Assoc 2016;5:e004237.

16. Miller CA, Corbin KD, da Costa KA, Zhang $S$, Zhao X, Galanko $J A$, et al. Effect of egg ingestion on trimethylamine- $\mathrm{N}$-oxide production in humans: a randomized, controlled, dose-response study. Am J Clin Nutr 2014;100:778-786.

17. Cho CE, Caudill MA. Trimethylamine-N-oxide: friend, foe, or simply caught in the cross-fire? Trends Endocrinol Metab 2017;28:121-130.

18. Boutagy $N E$, Neilson $A P$, Osterberg KL, Smithson AT, Englund TR, Davy BM, et al. Short-term high-fat diet increases postprandial trimethylamine-N-oxide in humans. Nutr Res 2015; 35:858-864.

19. Nie J, Xie L, Zhao BX, Li Y, Qiu B, Zhu F, et al. Serum trimethylamine $\mathrm{N}$-oxide concentration is positively associated with first stroke in hypertensive patients. Stroke 2018;49:20212028.

20. Yin J, Liao SX, He Y, Wang S, Xia GH, Liu FT, et al. Dysbiosis of gut microbiota with reduced trimethylamine-n-oxide level in patients with large-artery atherosclerotic stroke or transient ischemic attack. J Am Heart Assoc 2015;4:e002699.

21. Randrianarisoa $E_{1}$ Lehn-Stefan $A$, Wang $X$, Hoene $M$, Peter $A$, Heinzmann SS, et al. Relationship of serum trimethylamine $\mathrm{N}$-oxide (TMAO) levels with early atherosclerosis in humans. Sci Rep 2016;6:26745.

22. Svingen GF, Zuo H, Ueland PM, Seifert R, Løland KH, Pedersen $E R$, et al. Increased plasma trimethylamine- $\mathrm{N}$-oxide is associated with incident atrial fibrillation. Int J Cardiol 2018; 267:100-106.

23. Tang WH, Wang Z, Li XS, Fan Y, Li DS, Wu Y, et al. Increased trimethylamine $\mathrm{N}$-oxide portends high mortality risk inde- pendent of glycemic control in patients with type 2 diabetes mellitus. Clin Chem 2017;63:297-306.

24. Papandreou $C$, Bulló $M$, Zheng $Y$, Ruiz-Canela $M, Y u$, Guasch-Ferré $\mathrm{M}$, et al. Plasma trimethylamine-N-oxide and related metabolites are associated with type 2 diabetes risk in the Prevención con Dieta Mediterránea (PREDIMED) trial. Am J Clin Nutr 2018;108:163-173.

25. Fu $\mathrm{Q}$, Zhao M, Wang D, Hu H, Guo C, Chen W, et al. Coronary plaque characterization assessed by optical coherence tomography and plasma trimethylamine- $\mathrm{N}$-oxide levels in patients with coronary artery disease. Am J Cardiol 2016;118: 1311-1315.

26. Tan Y, Sheng Z, Zhou P, Liu C, Zhao H, Song L, et al. Plasma Trimethylamine $\mathrm{N}$-oxide as a novel biomarker for plaque rupture in patients with ST-segment-elevation myocardial infarction. Circ Cardiovasc Interv 2019;12:e007281.

27. Suzuki T, Heaney LM, Bhandari SS, Jones DJ, Ng LL. Trimethylamine $\mathrm{N}$-oxide and prognosis in acute heart failure. Heart 2016;102:841-848.

28. Kanitsoraphan $C$, Rattanawong $P$, Charoensri $S$, Senthong V. Trimethylamine $\mathrm{N}$-oxide and risk of cardiovascular disease and mortality. Curr Nutr Rep 2018;7:207-213.

29. Trøseid M, Ueland T, Hov JR, Svardal A, Gregersen I, Dahl CP, et al. Microbiota-dependent metabolite trimethylamine- $\mathrm{N}$-oxide is associated with disease severity and survival of patients with chronic heart failure. J Intern Med 2015;277:717-726.

30. Tang WH, Wang Z, Fan $Y$, Levison $B$, Hazen JE, Donahue LM, et al. Prognostic value of elevated levels of intestinal microbe-generated metabolite trimethylamine- $\mathrm{N}$-oxide in patients with heart failure: refining the gut hypothesis. J Am Coll Cardiol 2014;64:1908-1914.

31. Bain MA, Faull R, Fornasini G, Milne RW, Evans AM. Accumulation of trimethylamine and trimethylamine- $\mathrm{N}$-oxide in end-stage renal disease patients undergoing haemodialysis. Nephrol Dial Transplant 2006;21:1300-1304.

32. Missailidis $C$, Hällqvist J, Qureshi AR, Barany $P$, Heimbürger 0 , Lindholm B, et al. Serum trimethylamine-N-oxide is strongly related to renal function and predicts outcome in chronic kidney disease. PLoS One 2016;11:e0141738.

33. Kim RB, Morse BL, Djurdjev O, Tang M, Muirhead N, Barrett B, et al. Advanced chronic kidney disease populations have elevated trimethylamine $\mathrm{N}$-oxide levels associated with increased cardiovascular events. Kidney Int 2016;89:1144-1152.

34. Li D, Ke Y, Zhan R, Liu C, Zhao M, Zeng A, et al. Trimethylamine- $\mathrm{N}$-oxide promotes brain aging and cognitive impairment in mice. Aging Cell 2018;17:e12768.

35. Vogt NM, Romano KA, Darst BF, Engelman CD, Johnson SC, Carlsson $\mathrm{CM}$, et al. The gut microbiota-derived metabolite 
trimethylamine $\mathrm{N}$-oxide is elevated in Alzheimer's disease. Alzheimers Res Ther 2018;10:124.

36. Wang Z, Klipfell E, Bennett BJ, Koeth R, Levison BS, Dugar B, et al. Gut flora metabolism of phosphatidylcholine promotes cardiovascular disease. Nature 2011;472:57-63.

37. Gregory JC, Buffa JA, Org E, Wang Z, Levison BS, Zhu W, et al. Transmission of atherosclerosis susceptibility with gut microbial transplantation. J Biol Chem 2015;290:5647-5660.

38. Zhu W, Gregory JC, Org E, Buffa JA, Gupta N, Wang Z, et al. Gut microbial metabolite TMAO enhances platelet hyperreactivity and thrombosis risk. Cell 2016;165:111-124.

39. Koeth RA, Wang Z, Levison BS, Buffa JA, Org E, Sheehy BT, et al. Intestinal microbiota metabolism of L-carnitine, a nutrient in red meat, promotes atherosclerosis. Nat Med 2013;19: 576-585.

40. Seldin MM, Meng Y, Qi H, Zhu W, Wang Z, Hazen SL, et al. Trimethylamine $\mathrm{N}$-oxide promotes vascular inflammation through signaling of mitogen-activated protein kinase and nuclear factor-KB. J Am Heart Assoc 2016;5:e002767.

41. Sun $X$, Jiao $X, M a Y$, Liu $Y$, Zhang $L$, He $Y$, et al. Trimethylamine $\mathrm{N}$-oxide induces inflammation and endothelial dysfunction in human umbilical vein endothelial cells via activating ROS-TXNIP-NLRP3 inflammasome. Biochem Biophys Res Commun 2016;481:63-70.

42. Wang Z, Levison BS, Hazen JE, Donahue L, Li XM, Hazen SL. Measurement of trimethylamine- $\mathrm{N}$-oxide by stable isotope dilution liquid chromatography tandem mass spectrometry. Anal Biochem 2014;455:35-40.

43. Skagen K, Trøseid M, Ueland T, Holm S, Abbas A, Gregersen I, et al. The carnitine-butyrobetaine-trimethylamine- $\mathrm{N}$-oxide pathway and its association with cardiovascular mortality in patients with carotid atherosclerosis. Atherosclerosis 2016; 247:64-69.

44. Meyer KA, Benton TZ, Bennett BJ, Jacobs DR Jr, Lloyd-Jones DM, Gross MD, et al. Microbiota-dependent metabolite trimethylamine $\mathrm{N}$-oxide and coronary artery calcium in the coronary artery risk development in young adults study (CARDIA). J Am Heart Assoc 2016;5:e003970.

45. Ma J, Pazos IM, Gai F. Microscopic insights into the proteinstabilizing effect of trimethylamine N-oxide (TMAO). Proc Natl Acad Sci U S A 2014;111:8476-8481.

46. Collins HL, Drazul-Schrader D, Sulpizio AC, Koster PD, Williamson Y, Adelman SJ, et al. L-Carnitine intake and high trimethylamine $\mathrm{N}$-oxide plasma levels correlate with low aortic lesions in ApoE(-/-) transgenic mice expressing CETP. Atherosclerosis 2016;244:29-37.

47. Yoshida N, Yamashita T, Hirata KI. Gut microbiome and cardiovascular diseases. Diseases 2018;6:E56.

48. Chen J, Guo Y, Gui Y, Xu D. Physical exercise, gut, gut microbiota, and atherosclerotic cardiovascular diseases. Lipids Health Dis 2018;17:17.

49. Zhang $\mathrm{H}$, Meng J, Yu H. Trimethylamine N-oxide supplementation abolishes the cardioprotective effects of voluntary exercise in mice fed a western diet. Front Physio/ 2017;8:944.

50. Zhu W, Wang Z, Tang WHW, Hazen SL. Gut microbe-generated trimethylamine $\mathrm{N}$-oxide from dietary choline is prothrombotic in subjects. Circulation 2017;135:1671-1673.

51. Chen ML, Yi L, Zhang Y, Zhou X, Ran L, Yang J, et al. Resveratrol attenuates trimethylamine- $\mathrm{N}$-oxide (TMAO)-induced atherosclerosis by regulating TMAO synthesis and bile acid metabolism via remodeling of the gut microbiota. MBio 2016;7:e02210-e02215.

52. Kuka J, Liepinsh E, Makrecka-Kuka M, Liepins J, Cirule $H_{\text {, }}$ Gustina D, et al. Suppression of intestinal microbiota-dependent production of pro-atherogenic trimethylamine $\mathrm{N}$-oxide by shifting L-carnitine microbial degradation. Life Sci 2014; 117:84-92.

53. Wang $Z$, Roberts AB, Buffa JA, Levison BS, Zhu W, Org E, et al. Non-lethal inhibition of gut microbial trimethylamine production for the treatment of atherosclerosis. Cell 2015; 163:1585-1595. 